\title{
ANGPTL8 reverses established adriamycin cardiomyopathy by stimulating adult cardiac progenitor cells
}

\author{
Shuyuan Chen ${ }^{1}$, Jiaxi Chen ${ }^{2}$, Xing-Li Meng ${ }^{1}$, Jin-Song Shen ${ }^{1}$, Jing Huang ${ }^{3}$, Pintong \\ Huang $^{3}$, Zhaoxia Pu${ }^{3}$, Nathan H. McNeill ${ }^{1}$, Paul A. Grayburn ${ }^{1,4}$ \\ 1 Baylor Research Institute, Dallas, TX, USA \\ 2 the University of Texas Southwestern Medical Center at Dallas, Medical School, Dallas, TX, , USA \\ ${ }^{3}$ Department of Ultrasonography, The 2nd Affiliated Hospital of Zhejiang University College of Medicine, Hangzhou, Zhejiang \\ Province, China \\ ${ }^{4}$ Department of Internal Medicine, Division of Cardiology, Baylor Heart and Vascular Institute, Baylor University Medical \\ Center, Dallas, Texas, USA \\ Correspondence to: Paul A. Grayburn, email: paulgr@baylorhealth.edu \\ Keywords: Adriamycin cardiomyopathy, Angiopoietin-like protein 8, Ultrasound targeted microbubble destruction (UTMD), Pro- \\ genitor cells, Paired immunoglobulin like-receptor B (PirB) \\ Received: September 24, $2016 \quad$ Accepted: October 07, $2016 \quad$ Published: November 03, 2016
}

\section{ABSTRACT}

Established adriamycin cardiomyopathy is a lethal disease. When congestive heart failure develops, mortality is approximately $\mathbf{5 0 \%}$ in a year. It has been known that ANGPTLs has various functions in lipid metabolism, inflammation, cancer cell invasion, hematopoietic stem activity and diabetes. We hypothesized that ANGPTL8 is capable of maintaining heart function by stimulating adult cardiac progenitor cells to initiate myocardial regeneration. We employed UTMD to deliver piggybac transposon plasmids with the human ANGPTL8 gene to the liver of rats with adriamycin cardiomyopathy. After ANGPTL8 gene liver delivery, overexpression of transgenic human ANGPTL8 was found in rat liver cells and blood. UTMD- ANGPTL8 gene therapy restored LV mass, fractional shortening index, and LV posterior wall diameter to nearly normal. Our results also showed that ANGPTL8 reversed established ADM cardiomyopathy. This was associated with activation of ISL-1 positive cardiac progenitor cells in the epicardium. A time-course experiment shown that ISL-1 cardiac progenitor cells proliferated and formed a niche in the epicardial layer and then migrated into sub-epicardium. The observed myocardial regeneration accompanying reversal of adriamycin cardiomyopathy was associated with upregulation of PirB expression on the cell membrane of cardiac muscle cells or progenitor cells stimulated by ANGPTL8.

\section{INTRODUCTION}

Adriamycin (ADM) is one of the most widely prescribed and effective cytotoxic drugs used in oncology. However, the utility of adriamycin is limited by cumulative, dose- related, progressive myocardial damage that may lead to congestive heart failure (CHF). Thus, patients who may benefit from continued administration of the drug must withdraw from adriamycin therapy and switch to an alternative agent, which may be less effective [1]. An estimated cumulative percentage of $5 \%$ of adriamycin induced CHF patients at a cumulative dose of $400 \mathrm{mg} / \mathrm{m}^{2}, 26 \%$ of patients at $550 \mathrm{mg} / \mathrm{m}^{2}$, and
$48 \%$ of patients at $700 \mathrm{mg} / \mathrm{m}^{2}$. Although adriamycin cardiomyopathy accounts for only a small number of heart failure cases compared to ischemic cardiomyopathy or idiopathic dilated cardiomyopathy, it is a lethal disease. When congestive heart failure develops, mortality is approximately $50 \%$ in a year. Moreover, it often occurs in relatively young patients with curable malignancies such as breast cancer. Adriamycin is well known for its cardiac toxicity during chemotherapy for cancer. The cardiotoxicity is due to free radical formation and lipid peroxidation, which results in changes in lysosomes, sarcolemma's, mitochondria, and sarcoplasmic reticulum. These changes induce calcium overload, activation of 
hydrolytic enzyme, and reduction in energy production. Reduction of cardiac function was a result of the loss of structural integrity. Because adriamycin cardiotoxicity is dose dependent, it has been used to predictably induce heart failure in different animal species considered as a decent animal model with heart failure [2-6]. It will be very crucial to study how to prevent or cure adriamycin cardiomyopathy for these cancer patients. Traditional medical therapy for $\mathrm{CHF}$ is directed toward relief of symptoms and blockade of neurohormonal activation. In patients with advanced stages of CHF, heart transplant or left ventricular assist devices may be appropriate, but are expensive and have limited availability. The ideal goal for CHF therapy is myocardial regeneration. It is currently known that cardiac myocytes are capable of regeneration, via various mechanisms, including selfreplication of pre-existing adult cardiac muscle cells [7-8], differentiation of resident cardiac progenitor cells [9-10], dedifferentiation and proliferation of adult cardiac muscle cells [11-13] and transdifferentiation of fibroblast cells into cardiac muscle cells [14-15]. However, the magnitude of myocardial regeneration triggered by such mechanisms is small and it remains unclear whether myocardial regeneration in heart failure is sufficient to reverse established cardiomyopathy. Multiple clinical trials of stem cell therapy have been attempted but without clear improvement in cardiac function [16]. Part of this problem may be the fact that patients with advanced HF often have lack of blood supply to the heart and extensive scar tissue due to ischemic cardiomyopathy or longstanding non-ischemic cardiomyopathy with extensive fibrosis. The LIM homeodomain transcription factor (ISL-1 or Islet1) is a biomarker of cardiac progenitors or neural stem cells that give rise to the right ventricle, atria and outflow tract during cardiac development [17]. These cells can differentiate to the different cell types that form an adult heart (cardiomyocytes, smooth muscle cells, and endothelial cells) [18-19], However ISL-1 positive progenitor cells are usually quiescent during adult heart, being present in parasympathetic neurons, smooth muscle cells, a few cardiomyocytes within the proximal aorta and pulmonary artery and more abundantly in the sinoatrial node. The epicardium contains epicardium-derived cells (EPDCs), which undergo epithelial-to-mesenchymal transition (EMT) and differentiate into all main cell lineages of the heart [20]. However some evidence shows that adult EMCs primarily undergo fibrogenic EMT upon cardiac stress, such as hypertension or infarction, to generate myofibroblast-like cells [21]. These cells can contribute to the development of cardiac disease, such as fibrosis, potentially leading to impaired cardiac performance and arrhythmias, including sudden death. It is very crucial to find out the molecular and cellular aspects of these EMCs as well as associated factors, and their implication in EMT and cardiac fibrosis in order to prevent or treat certain heart diseases.
Angiopoietin-like proteins are a family of 8 member proteins that are structurally similar to the angiopoietins, all containing an amino-terminal coiled-coil domain, a linker region, and a carboxy-terminal fibrinogenlike domain except ANGPTL8. ANGPTLs have various functions in lipid metabolism, inflammation, cancer cell invasion, hematopoietic stem activity. ANGPTL8 (also known as C19orf80) is a 596 bp cDNA with a short sequence in the $\mathrm{N}$-terminus that is highly enriched in the liver and adipocytes [22-27]. Yi et al [28-29] found that a hepatic overexpression of ANGPTL8 after a hydrodynamic injection of non-viral sleeping beauty transposon plasmids into the tail vein of adult mice had several effects: it stimulated the proliferation of pancreatic beta cells, increased insulin secretion, improved glucose tolerance and expanded the mass of the beta cell islets. We have confirmed the pancreatic beta cell proliferation induced by $A N G P T L 8$, but it was not able to reverse diabetes in rats with STZ- induced diabetes [30] or obese rhesus monkey with naturally occurring diabetes [data not shown]. To our knowledge, the effects of ANGPTL8 on myocardial regeneration in heart failure have not been reported. Our group developed a novel approach to gene therapy by targeting the delivery of non-viral DNA to the organs using ultrasound-targeted microbubble destruction (UTMD) [31-37]. In this study, we employed UTMD to deliver the $A N G P T L 8$ gene to the liver of rats with established cardiomyopathy induced by adriamycin. We found that hepatic overexpression of ANGPTL8 can reverse established cardiomyopathy.

\section{RESULTS}

\section{UTMD targeting of human ANGPTL8 gene to liver of rats}

Figure 1 shows that human ANGPTL8 signal was detected in liver cells after UTMD-pXL-BASII-CIANGPTL8/pCI-hyPB delivered to the liver (Figure 1D) but was not detected in normal rat liver, nor in the controls treated with ADM only and ADM plus UTMD-DsRed reporter gene (Figure 1A-1C). Human ANGPTL8 signal was detected in the cytoplasm of liver cells of rats from 3 days to 4 weeks after delivery of the ANGPTL8 gene to liver by UTMD. Figure 1E shows fasting plasma levels of human ANGPTL8 in rats. In the normal and DsRed reporter gene control rat groups, no human ANGPTL8 was detected in fasting plasma. However, in the treatment groups, human ANGPTL8 was detected in fasting plasma from day 1 to day 28 after UTMD. We further evaluated human ANGPTL8 mRNA levels using qRT-PCR. The results (Figure 1F) showed that human ANGPTL8 mRNA levels after UTMD-ANGPTL8 gene delivery were $25 \pm$ 8 fold greater, respectively, than for the normal, ADM 
only and ADM plus UTMD-DsRed control groups $(\mathrm{p}<0.001)$. Western blotting was employed to detect human ANGPTL8 from liver protein extracts, and the results showed that the human ANGPTL8 signal existed in the liver protein extracts after UTMD-ANGPTL8 gene delivery (data not shown).

\section{Reversal of established adriamycin cardiomyopathy after UTMD-ANGPTL8 gene therapy}

We decided to evaluate if there is any possible pharmacological effects of ANGPTL8 on rat hearts with established adriamycin cardiomyopathy. We employed echocardiography to evaluate heart structure and function in all groups. Figure $1 \mathrm{H}-\mathrm{K}$ demonstrated $\mathrm{M}$-mode images derived from 2D parasternal short axis views of the left ventricle showing decreased LV fractional shortening and LV mass in adriamycin cardiomyopathy with restoration to normal values by UTMD-ANGPTL8 gene therapy (Figure $1 \mathrm{~L}$ and $\mathrm{M}$ ).
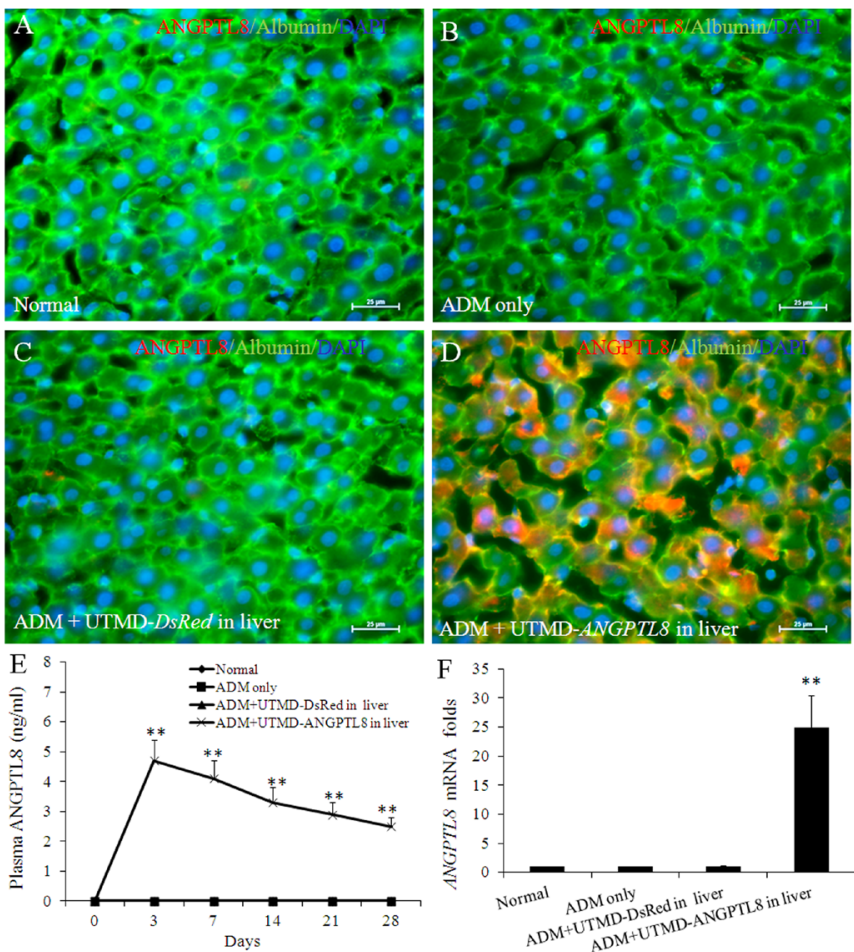

ANGPTL8 significantly upregulated PirB expression on membrane of cardiac muscle cells

Zheng, et al [38] reported that Paired immunoglobulin-like receptor $\mathrm{B}$ (PirB) in rodents or LILRB2 in human is a specific receptor of ANGPTLs in membrane of hematopoietic stem cells. ANGPTLs stimulate expansion of hematopoietic stem cells ex vivo by activating PirB. It also is an inhibitory receptor in neurons that inhibited regeneration of adult neuron cells [39]. However, there are no reports of PirB in adult cardiac muscle cells. Figure 2 shows robust presence of PirB on the surface of cTNT positive cardiac muscle cells (Figure 2A). However, under established cardiomyopathy induced by ADM, PirB was rarely seen (Figure 2B-C), after reversal of ADM cardiomyopathy by ANGPTL8 gene liver delivery, PirB was again seen on the surface of membrane of cardiac muscle cells (Figure 2D). We quantified PirB mRNA levels using qRT-PCR. The results (Figure 2E) showed that PirB mRNA levels are $89 \pm 16$, $12 \pm 4,16 \pm 6$, and $82 \pm 24$ folds respectively in normal rat heart, ADM only control heart, ADM plus UTMD-DsRed

G
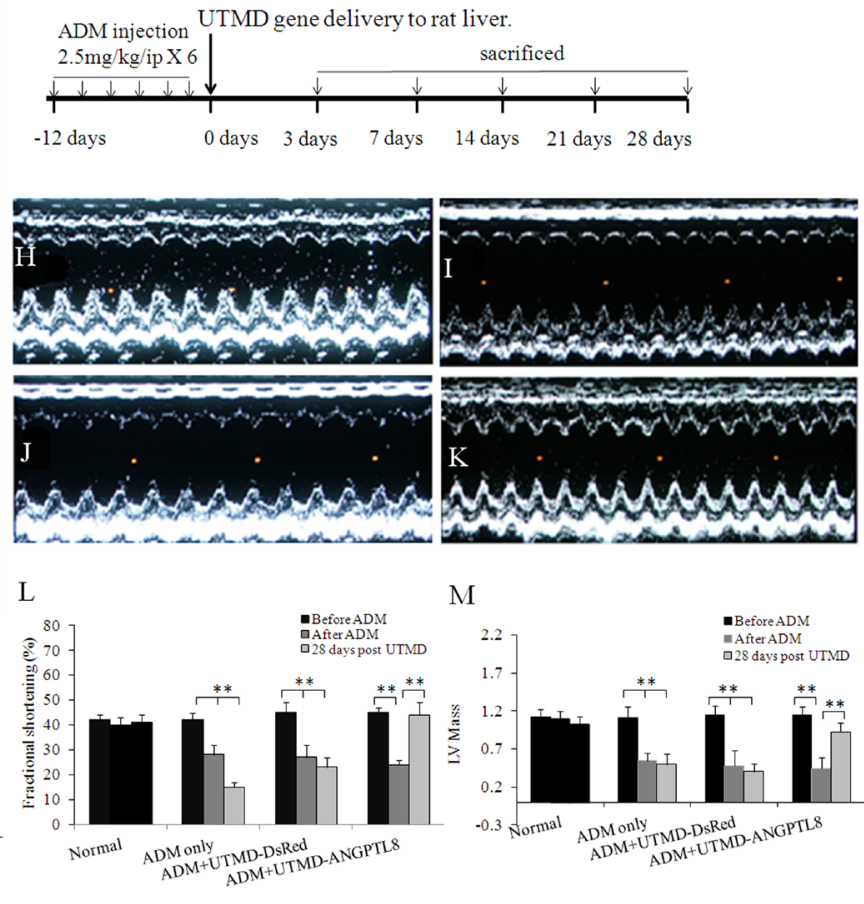

Figure 1: UTMD delivered ANGPTL8 gene to liver. (A-D) triple staining with ANGPTL8/Albumin/DAPI for normal rat liver (A), liver of ADM only rat (B), ADM plus UTMD-pXL-BASII-CI-DsRed/hyPB targeted to rat liver (C), ADM plus UTMD- pXL-BASIICI-ANGPTL8/pCI-hyPB targeted to liver (D). Scale bar is $25 \mu \mathrm{m}$. (E) a graphic of fasting plasma human ANGPTL8 levels. In normal, ADM control and ADM plus UTMD-DsRed groups, human ANGPTL 8 was not detectable. Values are presented as mean \pm SEM. $n=6$ per group; $* * \mathrm{P}<0.001$ vs control groups. (F) a graphic of RT-qPCR for human ANGPTL 8 mRNA, housekeeping gene is actin gene. Values are presented as mean \pm SEM. $n=6$ per group; $* * \mathrm{P}<0.001 v s$ control groups. (G) Schematic indicating time-course experiment. (H-K) Results of echocardiographic measurement of cardiac structure and function. M-mode images at 2D parasternal short axis of the left ventricle, (H) normal rat heart, (I) ADM control rat heart, (J) ADM plus UTMD-DsRed rat heart, (K) ADM plus UTMD-ANGPTL8 rat heart. (L) a graphic of fractional shortening, $(\mathbf{M})$ a graphic of LV mass, Values are presented as mean \pm SEM. $n=6$ per group; ${ }^{* *} \mathrm{P}<0.001 v s$ after $\mathrm{ADM}$. 
heart, and ADM plus UTMD-ANGPTL8 heart groups. The results of western blotting shows that PirB existed in all the heart protein extracts, was significantly deceased in ADM treated heart samples, and recovered after UTMDANGPTL8 gene delivery (Figure 2F). We decided to use cultured mouse HL-1 atrial cardiomyocyte cells to confirm what we found in vivo experiment, Figure $3 \mathrm{~A}$ shown that PirB clearly localized on the membrane of HL-1 atrial cardiomyocyte cells as a membrane receptor, and exogenously ANGPTL8 peptide are binding with PirB on the membrane of HL-1 atrial cardiomyocyte cells (Figure 3B-D).
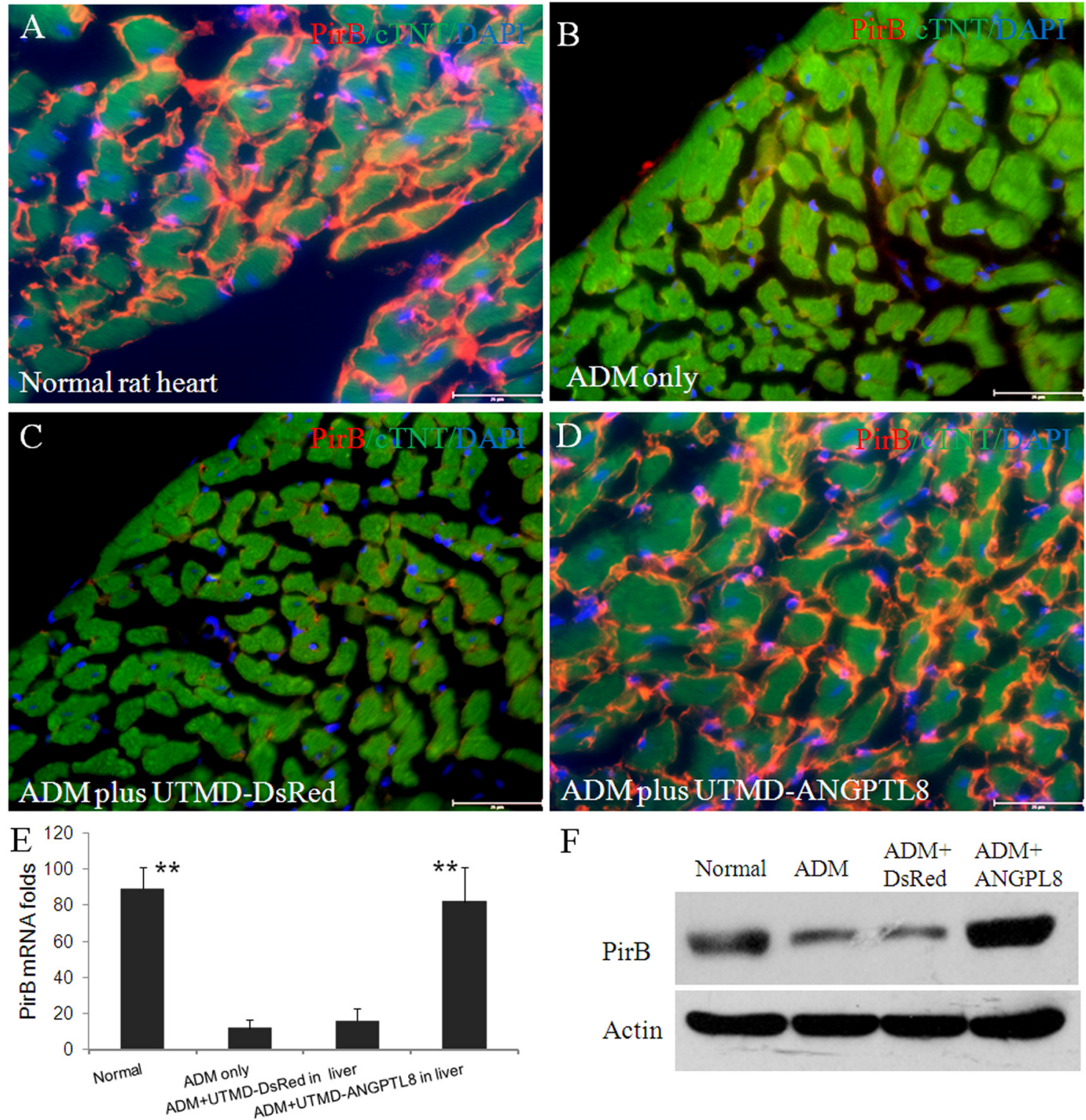

$\mathrm{F}$

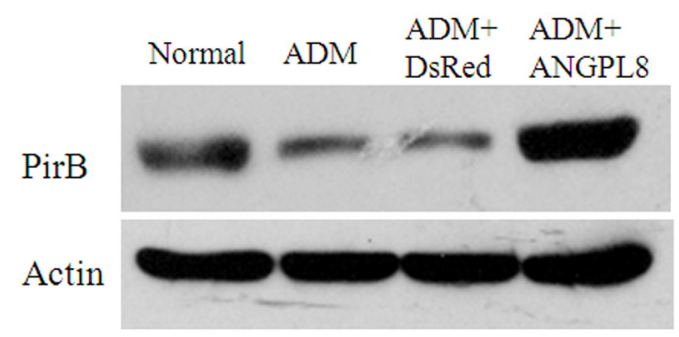

Figure 2: Upper panel (A-D): Paired immunoglobulin-like receptor B (PirB) distributed on membrane of cardiac muscle cells of adult rat. (A) Normal rat heart; (B) ADM only control heart; (C) ADM plus UTMD-DsRed heart; (D) ADM plus UTMD-ANGPTL8 heart; Scale bar is $25 \mu \mathrm{m}$. Red color for PirB signal; green for cardiac troponin T signal; blue for nucleus identity. (E) a graphic of RT-qPCR for PirB mRNA, housekeeping gene is actin gene. Values are presented as mean \pm SEM. $n=6$ per group; ${ }^{*} \mathrm{P}<0.001$ vs ADM only and ADM plus DsRed groups. (F) Western blots detecting PirB from protein extracts of rat heart tissue, Actin is a marker of cytoplasm proteins. 
$(\mathrm{p}<0.001)$ (Figure 4E). The results of western blotting shown that ISL-1 signal was scant in nuclear protein extracts of normal heart, ADM only and ADM plus UTMD-DsRed heart samples, but significantly increased in after UTMD-ANGPTL8 gene delivery(Figure 4K). Finally, the location of ISL-1 positive cells was confined to the epicardium and sub-epicardium (Figure 4I) and not seen in endocardium and myocardium (Figure $4 \mathrm{G}-\mathrm{H}$ and J) $(\mathrm{P}<0.001)$.

We decided further to investigate the effect of ANGPTL 8 on ISL-1 positive cardiac progenitor cells with a time-course experiment. Figure 5 shows that no ISL-1 signal was seen in epicardial layer cells before UTMDANGPTL8 gene delivery (Figure 5A), but was observed 3 days post UTMD- ANGPTL8 treatment (Figure 5B). ISL-1 positive cells appeared to cluster into epicardial niches of cardiac progenitor cells 7 days post UTMD- ANGPTL8 gene delivery (Figure 5C), further proliferating and migrating into sub-epicardial myocardium (Figure 5D-E) and finally differentiating into new cardiac muscle cells (Figure 5F).

\section{Regenerating cardiac muscle cells are in proliferation}

We used a mitotic marker (anti-phospho-histone H3 (Ser10) (PHH3)) to demonstrate if regenerating cardiac muscle cells were proliferating. We calculated the percentage of $\mathrm{PHH} 3$ positive cardiomyocytes by counting stained nuclei (pink color) from $1000 \mathrm{cTnT}$
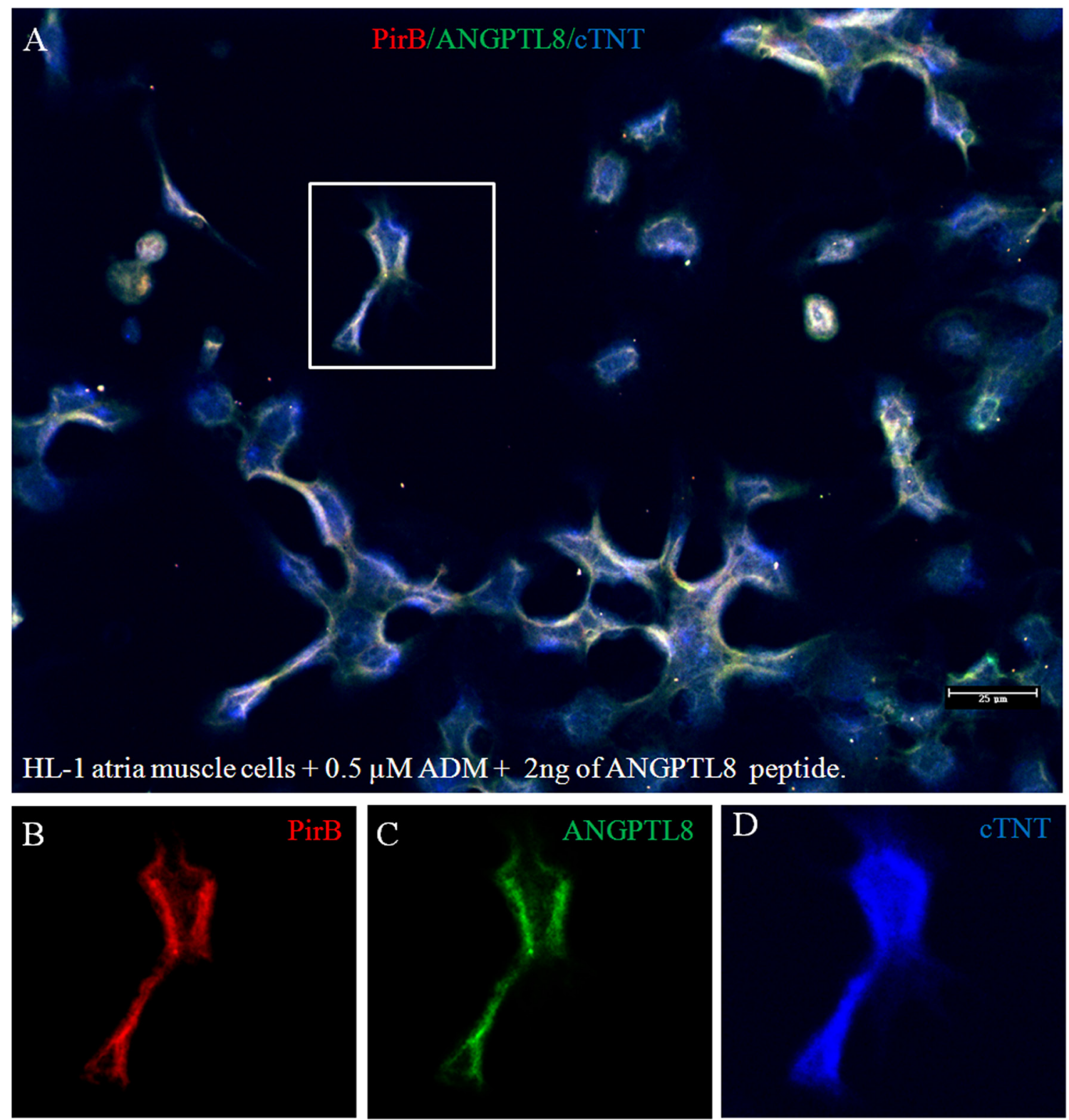

Figure 3: The culture atria muscle cell line (HL-1) treated with $0.5 \mu$ M ADM plus 2 ng of ANGPTL8 peptide. Scale bar is $25 \mu \mathrm{m}$. Red color for PirB signal; green for ANGPTL8 signal; blue for cardiac troponin T. 
positive cardiomyocytes cells in the sub-epicardium using serial sections through each rat heart ( $\mathrm{n}=6$ each group). Figure 6 shows that PHH3 signal was observed within the nuclear positive cells by confocal microscopy in ADM plus UTMD- ANGPTL8 groups. The percentage of PHH3 positive sub-epicardial muscle cells in the rats treated with UTMD- ANGPTL 8 gene therapy 28 days postUTMD (Figure $6 \mathrm{~F}$ ) was $3.8 \pm 0.60 \%$ ( $<<0.001$ vs controls). Figure 6D-E shows evidence of cell proliferation ( $\mathrm{PHH} 3$ expression) confined to the epicardial layer. The western blotting results showed that $\mathrm{PHH} 3$ signal was not seen in the adult heart nuclear protein extracts except in the ADM plus UTMD-ANGPTL8 gene delivery hearts (Figure 6H).

\section{ANGPTL8 protein therapy also reversed established ADM cardiomyopathy}

We wonder if exogenous ANGPTL8 protein delivery has the same effects as its gene liver delivery. We set three different dosage of $A N G P T L 8$ protein for intramuscular injection daily for 14 days; Supplemental Figure 1A shows fasting plasma levels of human ANGPTL8 protein in rats. No human ANGPTL 8 was detected in fasting plasma in
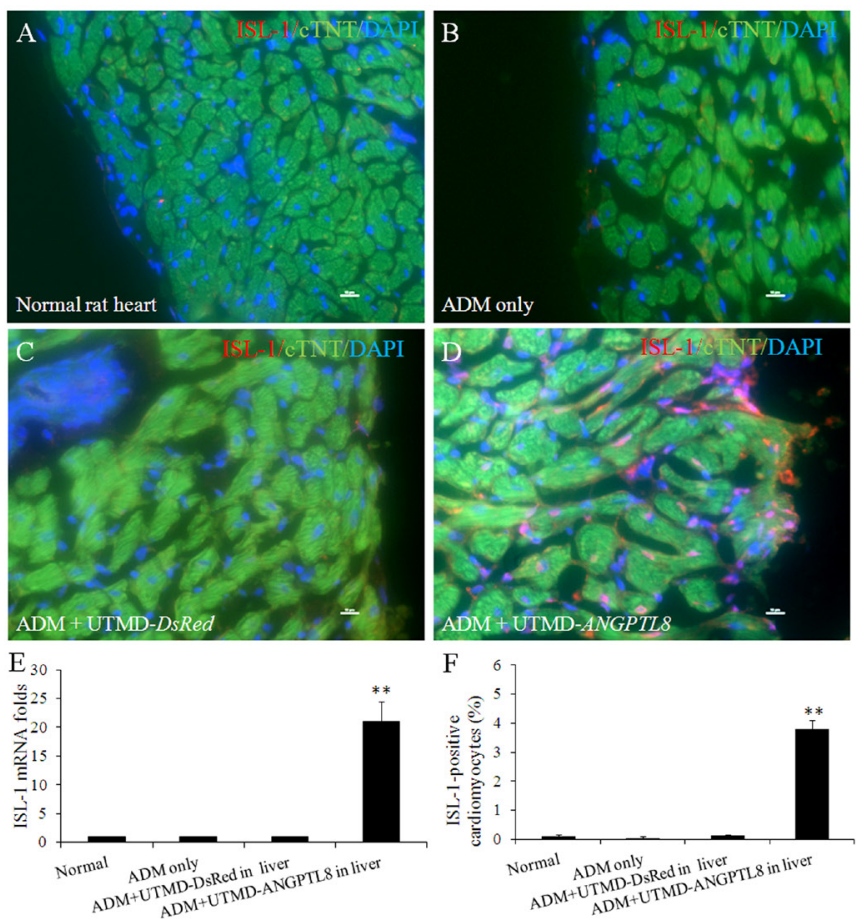

ADM plus saline injection, in the three different dosages groups. However, human ANGPTL8 was detected in fasting plasma from day 3, 7, 10 and 14 during daily injection of $A N G P T L 8$ protein for consistently 14 days. Supplementary Figure 1B-C demonstrated M-mode images derived from $2 \mathrm{D}$ parasternal short-axis views of the left ventricle, compared with normal rat heart, ADM plus saline injection control rat heart, ADM plus $A N G P T L 8-5 \mu \mathrm{g} / \mathrm{kg}$ rat heart, ADM plus $A N G P T L 8-20 \mu \mathrm{g} /$ $\mathrm{kg}$ rat heart and ADM plus $A N G P T L 8-40 \mu \mathrm{g} / \mathrm{kg}$ rat heart. There was decreased LV fractional shortening and LV mass in adriamycin cardiomyopathy with some degree of restoration by $A N G P T L 8$ protein therapy. Supplementary Figure 2 shows that ISL-1 positive cells in epicardial layer were activated by $A N G P T L 8$ protein injection with a dose dependent manner after 14 days treatment. Supplementary Figure 3 displayed that some epicardial cells and subepicardial cardiac muscle cells are in proliferation after ANGPTL8 protein therapy.

\section{DISCUSSION}

Balmer, et al [40] reported that cardiac progenitor cells in epicardial layer of adult heart are a resource for

Figure 4: The activation of ISL-1 (an early cardiac muscle differentiation marker) in epicardium and sub-epicardial layer after ADM plus UTMD-ANGPTL8. (A) normal rat heart, (B) ADM control rat heart, (C) ADM plus UTMD-DsRed, (D) ADM plus UTMD-ANGPTL8. Scale bar is $10 \mu \mathrm{m}$. (E) a graphic of qRT-PCR for ISL-1 mRNA, housekeeping gene is actin gene. Values are presented as mean \pm SEM. $n=6$ per group; $* * \mathrm{P}<0.001$ vs control groups. (F) a graphic for percentage of ISL-1-positive cardiomyctes cells. Values are presented as mean \pm SEM. $n=6$ per group; ${ }^{* *} p<0.001$ vs control groups. The distribution of ISL-1-positive adult cardiac progenitor cells after ADM plus UTMD-ANGPTL8. (G) Endocardium, (H) Myocardium, (I) Sub-epicardium, at hearts of ADM plus UTMD-ANGPTL8 group. Scale bar is $100 \mu \mathrm{m}$. (J) a graphic for percentage of ISL-1-positive cardiomyoctes cells. Values are presented as mean \pm SEM. $n=6$ per group; $* * p<0.001$ vs endocardium and myocardium. (K) Western blots detecting ISL-1 from nuclear protein extracts of rat heart tissue, Lamin is a marker of nuclear protein. 
myocardial regeneration and they are able to differentiate into cardiac muscle cells, coronary artery muscle cells and vascular endothelial cells. They appear to originate from migration of hematopoietic stem cells from bone marrow. Under some pathological states, these cells may differentiate into fibroblasts, forming scar tissue in heart. It will be a crucial to study how to drive these cardiac progenitor cells to differentiate into muscle cells but not fibroblasts. There is some evidence that ANGPTL1-7 expands hematopoietic stem cells ex vivo and stimulates the formation of niches of hematopoietic cells in bone marrow. Our study demonstrates that ANGPTL8 can stimulate adult myocardial regeneration by proliferation of cardiac progenitor cells located at epicardium. Timecourse experiments suggest that ANGPTL 8 drove the differentiation of cardiac progenitor/stem cells into ISL-1 positive cardiac progenitor cells which formed niches and migrated into sub-epicardial myocardium. It still is not clear what the relationship is between WT1 positive progenitor cells and ISL-1 cardiac progenitor cells in epicardium. The exact molecular mechanism by which $A N G P T L 8$ activated these quiescent adult cardiac
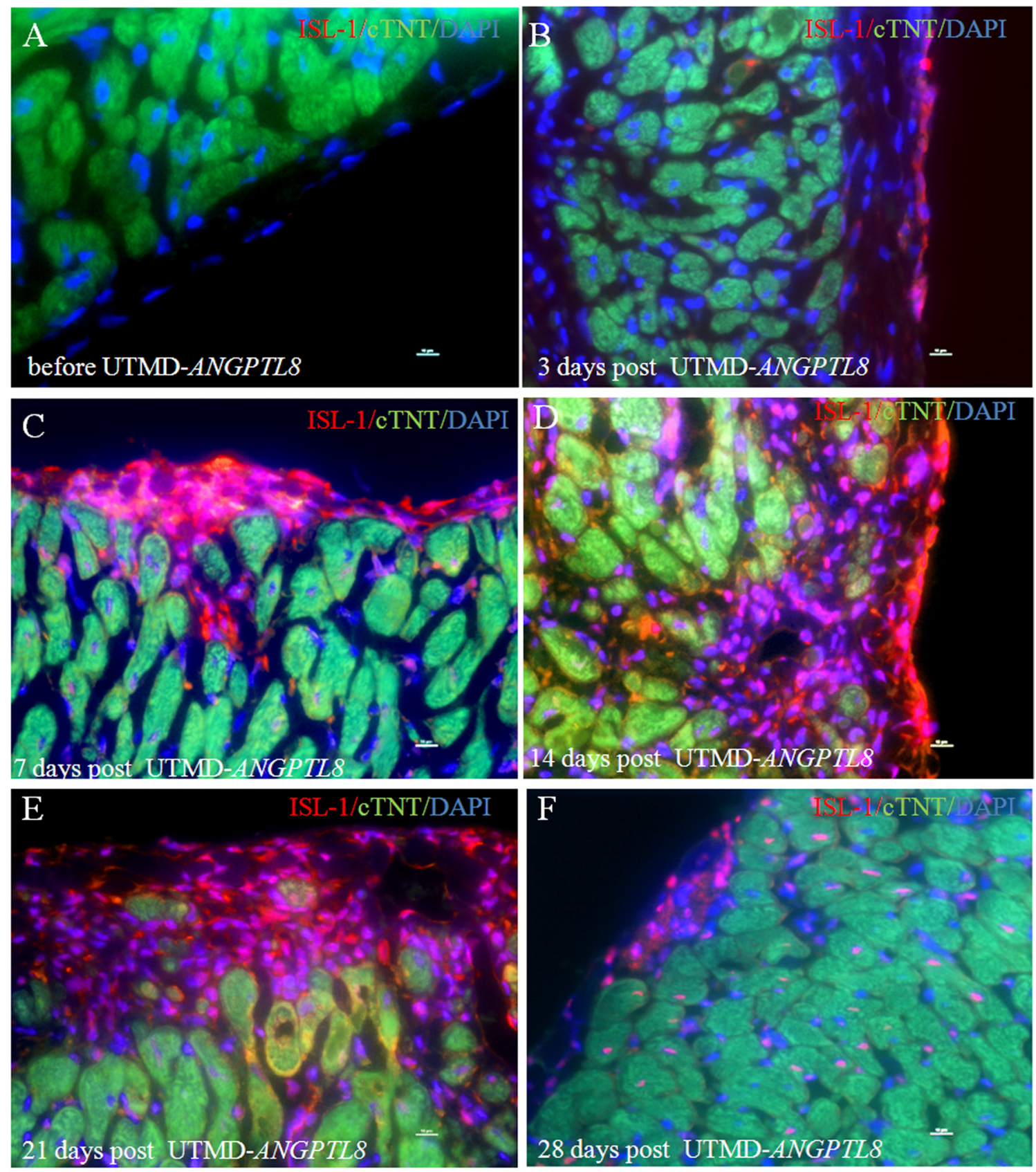

Figure 5: A time-course experiment show that ANGPTL8 induced epicardial layer cells (A) into ISL-1-positive cardiac progenitor cells $(\mathbf{B})$ and formed niches of cardiac progenitor cells in epicardium $(\mathbf{C})$ proliferated and migrated into myocardium layer (D-E) and differentiate into new cardiac muscle cells $(\mathbf{F})$. Scale bar is $10 \mu \mathrm{m}$. 

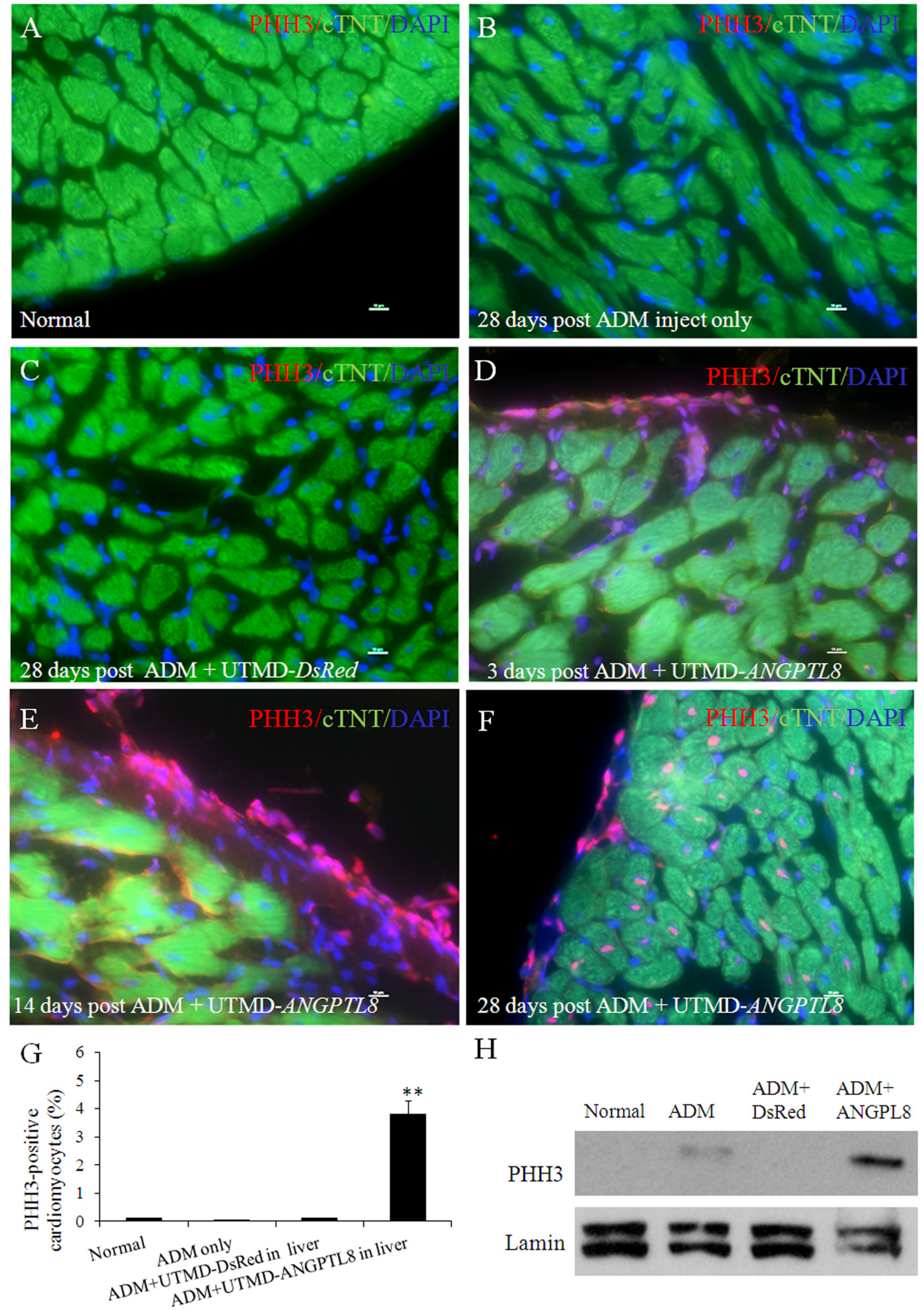

$\mathrm{H}$

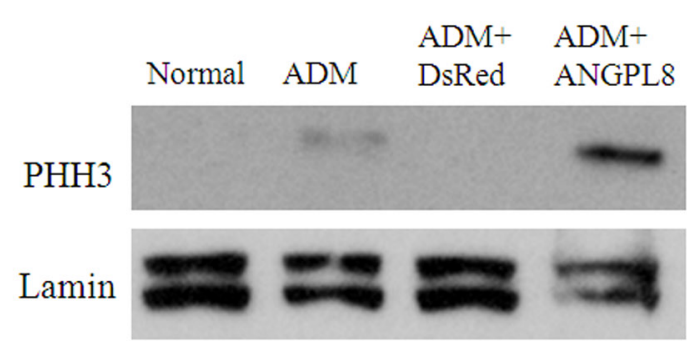

Figure 6: Phospho-histone H3 (PHH3) staining shown some regenerating cardiac muscle cells are in proliferation. (A) normal rat heart, (B) 28 days post ADM inject, (C) 28 days post ADM plus UTMD-DsRed, (D) 3 days post ADM plus UTMD-ANGPTL8. (E) 14 days post ADM plus UTMD-ANGPTL8. (F) 28 days post ADM plus UTMD-ANGPTL8. Scale bar is $10 \mu \mathrm{m}$, (G) Percentage of PHH3-positive cardiomyctes cells. Values are presented as mean \pm SEM. $n=6$ per group; ${ }^{* *} p<0.001$ vs control groups. (H) Western blots detecting PHH3 from nuclear protein extracts of rat heart tissue, Lamin is a marker of nuclear protein. 
progenitor cells in adult hearts with established ADM cardiomyopathy is also not known.

Zheng, et al [38, 41-43] showed that the human immune inhibitory receptor leukocyte immunoglobulinlike receptor B2 (LILRB2) and its mouse ortholog paired immunoglobulin-like receptor (PirB) are receptors for several angiopoietin-like proteins. LILRB2 and PirB are expressed on human and mouse hematopoietic stem cells, respectively, and the binding of ANGPTLs to these receptors supported ex vivo expansion of hematopoietic stem cells. In mouse transplantation acute myeloid leukemia models, a deficiency in intracellular signaling of PirB resulted in increased differentiation of leukemia cells, revealing that PirB supports leukemia development. Their study indicated an unexpected functional significance of classical immune inhibitory receptors in maintenance of normal adult stem cells and in support of cancer development. Supplemental Figure 4 show that PirB exists on the surface membrane of ISL-1 positive cardiac progenitor cells. Our study suggests that ANGPTL8 expands cardiac progenitor cells in epicardium by the activation of PirB signal pathway. Although some data indicates that this inhibitory receptor signal is related to the Notch1 control pathway [44-46], further studies are needed to decipher the role of PirB in adult cardiac muscle cells under physiological or pathological states. Nevertheless, our finding that ANGPTL8 reverses established ADM induced cardiomyopathy offers hope that pathways exist for myocardial regeneration in adult animals. In conclusion, ANGPTL8 has previously unrecognized effects that result reversal of established cardiomyopathy by activation of PirB receptor on the cell membrane of resident adult cardiac progenitor cells. Further evaluation of the mechanism of the effect of ANGPTL8 on cardiac PirB receptors is needed.

\section{MATERIALS AND METHODS}

\section{Animal protocols}

Animal studies were performed according to National Institutes of Health $(\mathrm{NIH})$ recommendations and approved by our institutional animal research committee. Adult male Sprague-Dawley rats were purchased from Harlan Laboratories (Indianapolis, IN, USA).

The protocol was planned to test the hypothesis that delivery of ANGPTL8 by UTMD could reverse established adriamycin (ADM) cardiomyopathy defined as a fractional shortening $<30 \%$ by echocardiography after injection of ADM [47] at total dose of $15 \mathrm{mg} / \mathrm{kg} / \mathrm{ip}, 2.5 \mathrm{mg} /$ $\mathrm{kg} / \mathrm{ip} 6$ times over 2 weeks. Roughly $70 \%$ of animals developed an established cardiomyopathy after 6 doses of ADM. Only the animals with a fractional shortening $<30 \%$ were selected into the protocols.

\section{Protocol-1 for ANGTPL8 gene therapy}

There were 120 rats divided into three control groups of 30 rats each and a treatment group of 30 rats: (1) normal control rats; (2) ADM injection only; (3) ADM plus UTMD with a DsRed reporter gene (pXL-BASII-CIDsRed/pCI-hyPB); (4) ADM plus UTMD with ANGPTL8 (pXL-BASII-CI-ANGPTL8/pCI-hyPB). UTMD day was set as day 0 , all rats were euthanized at 3 days, 7 days, 14 days, 21 days and 28 days after UTMD. The thymidine analog, 5-bromo-2-deoxyuridine (BrdU) (100mg/kg) was injected intraperitoneally 6 hours prior to euthanizing.

Male Sprague-Dawley rats (230-270g) were anesthetized with intraperitoneal ketamine $(60 \mathrm{mg} /$ $\mathrm{kg}$ ) and xylazine $(5 \mathrm{mg} / \mathrm{kg})$, and a polyethylene tube (PE 50, Becton Dickinson, Franklin Lakes, TN, USA) was inserted into the right internal jugular vein by cutdown. Piggybac transposon donor plasmids and helper plasmids ratio (pXL-BASII-CI-ANGPTL8/pCI-hyPB) was 5:1. Microbubble or control solutions $(0.5 \mathrm{ml}$ diluted with $0.5 \mathrm{ml}$ phosphate-buffered solution (PBS)) were infused over 1 min 30 seconds via pump (Genie, Kent Scientific, Torrington, CT). During the infusion, ultrasound was directed to the liver using a commercially available ultrasound transducer (S3, Sonos 5500, Philips Ultrasound, Bothell, WA). Ultrasound was then applied in ultraharmonic mode (transmit 1.3 MHz/receive 3.6 $\mathrm{MHz}$ ) at a mechanical index of 1.2. Four bursts of ultrasound were triggered to every fourth end-systole by electrocardiogram using a delay of 45-70 ms after the peak of the $\mathrm{R}$ wave. These settings have shown to be optimal for plasmid delivery by UTMD using this instrument [48]. Bubble destruction was visually apparent in all rats. After UTMD, the jugular vein was tied off, the skin closed, and the animals allowed to recover. All of rats were euthanized using an overdose of sodium pentobarbital $(120 \mathrm{mg} / \mathrm{kg})$.

\section{Protocol-2 for ANGTPL8 protein therapy}

15 rats divided into (1) normal control rats; (2) ADM plus saline injection rats; (3) ADM plus ANGPTL8 protein at dose of $5 \mu \mathrm{g} / \mathrm{kg} /$ day intramuscular injection (into the right quadriceps) for 14 days; (4) ADM plus ANGPTL8 protein at dose of $20 \mu \mathrm{g} / \mathrm{kg} /$ day intramuscular injection for 14 days; (5) ADM plus ANGPTL8 protein at dose of $40 \mu \mathrm{g} / \mathrm{kg} /$ day intramuscular injection for 14 days. All rats were euthanized at 14 days after starting ANGTPL8 protein injection. The thymidine analog, 5-bromo-2-deoxyuridine (BrdU) (100mg/kg) was injected intraperitoneally 6 hours prior to euthanasia. Human recombinant protein ANGTPL8 protein was purchased from Phoenix Pharmaceuticals (Cat \# 056-61, Burlingame, CA, USA). 


\section{Manufacture of plasmid-containing lipid- stabilized microbubbles}

Lipid-stabilized microbubbles were made as previously described [48]. Briefly, $250 \mathrm{mg}$ of 1,2-dipalmitoyl-sn-glycero-3-phosphocholine, $50 \mathrm{mg}$ of 1,2-dipalmitoyl-sn-glycero-3-phosphoethanolamine and $10 \%$ glucose were mixed with PBS and boiled in water until fully dissolved. Next $2 \mathrm{mg}$ of plasmid DNA was mixed with $0.5 \mathrm{ml}$ of ethyl alcohol and centrifuged at $10,000 \mathrm{~g}$ for $5 \mathrm{~min}$. The supernatant fraction was removed, and the DNA pellet was placed in an incubator at $37^{\circ} \mathrm{C}$ for $5 \mathrm{~min}$ to remove any remaining ethyl alcohol. The DNA was then added to $50 \mu$ lof Lipofectamine 2000 (1 $\mathrm{mg} / \mathrm{ml}$; Invitrogen, Carlsbad, CA, USA) and mixed for $20 \mathrm{~min}$. This mixture was added to $250 \mu \mathrm{l}$ of liposome solution, $5 \mu \mathrm{l}$ of $10 \%$ albumin and $50 \mu \mathrm{l}$ of glycerol in 1.5 $\mathrm{ml}$ vials, and placed on ice. The headspace of the vials was filled with perfluoropropane gas, and the vials were then shaken for 30 seconds at $4^{\circ} \mathrm{C}$. The mean diameter and concentration of the microbubbles were $1.9 \pm 0.2$ $\mu \mathrm{m}$ and $5.2 \pm 0.3 \times 10^{9}$ bubbles per $\mathrm{ml}$, respectively. The concentration of plasmid carried by the microbubbles was $250 \pm 10 \mu \mathrm{g} / \mathrm{ml}$.

\section{Plasmid constructs}

Human ANGPTL8 cDNA with Sal1/Not1 cutting sites (Integrated DNA Technologies, Coralville, IA, USA) was subcloned into PiggyBac transposon plasmids (pXL-BSII donor plasmid) provided by Dr. Fraser at the University of Notre Dame (Notre Dame, IN, USA) [49], and hyperactive piggyBac transposase helper plasmid was provided by Dr. Bradley at Wellcome Trust Sanger Institute (Cambridge, UK) [50]. Cloning, isolation and purification of the plasmids were performed by standard procedures, and the PCR products were sequenced to confirm that no artefactual mutations were present.

\section{Immunohistochemistry}

Tissue samples were fixed in $10 \%$ formalin for 24 hours and transferred into $70 \%$ alcohol for paraffin embedding and $4 \%$ paraformaldehyde and $20 \%$ sucrose overnight at $4{ }^{\circ} \mathrm{C}$ for frozen sections. Cryostat sections $5-8 \mu \mathrm{m}$ in thickness were further fixed with acetone $\left(-20^{\circ} \mathrm{C}\right)$ for $5 \mathrm{~min}$ and quenched for $5-20 \mathrm{~min}$ with 10 $\mathrm{mM}$ glycine in PBS. Sections were then rinsed in PBS 3 times, and permeabilized with $0.5 \%$ Triton X-100 in PBS for $15 \mathrm{~min}$. The slides that needed further nuclear protein retrieval were subjected to boiling citrate buffer solution with tween 20 at $\mathrm{pH} 6.0$ for 5 minutes. Sections were blocked with Aquablock solution (EastCoast Bio, North Berwick, ME) at room temperature for $1 \mathrm{hr}$ and washed with PBS 1 time. The primary antibodies rat anti-PirB, 1: 200 dilution ( BD biosciences, San Jose, CA ), rabbit antiNotch1, 1:200, rabbit anti- FoxO1, 1:250, and rabbit antiISL-1, 1: 500 dilution, and mouse anti-cardiac troponin T, 1: 250 dilution, rabbit anti-PHH3, 1:200 dilution, rabbit anti-human ANGPTL8 at 1:100 dilution, rabbit anti-albumin, 1:250 dilution (Abcam Inc, Cambridge, MA), mouse anti-human ANGPTL8 1:200 dilution (Phoenix Pharmaceuticals, Burlingame, CA, USA), were added and incubated for $2 \mathrm{hrs}$ at RT or overnight at 4 ${ }^{\circ} \mathrm{C}$. After washing with PBS three times for $5 \mathrm{~min}$, the secondary antibody (Sigma, St; Louis, MO) anti-mouse $\lg \mathrm{G}$ conjugated with FITC; anti-rabbit IgG-conjugated with Texas Red, or anti-donkey $\lg G$ conjugated with Cy5) (1:250 dilution in block solution) were added and incubated for $1 \mathrm{hr}$ at RT. Sections were rinsed with PBS for $10 \mathrm{~min}, 3$ times, and incubated with Dapi (Invitrogen, Carlsbad, CA), 1: 5000 dilution for 5 min and washed 3 times with PBST, then mounted. A confocal microscope was used to take pictures.

\section{Culture of HL-1 Atria Muscle Cells line.}

The HL-1 cell line was a generous gift from Dr. William C. Claycomb [51] in Louisiana State University Medical Center, New Orleans, LA. The cells were maintained in Claycomb basal medium (Sigma) supplemented with $10 \%$ fetal bovine serum, $0.1 \mathrm{mM}$ norepinephrine and $2 \mathrm{mM} \mathrm{L}$-glutamine. HL-1 cells were treated with adriamycin at $0 \mu \mathrm{mol} / \mathrm{L}, 0.25 \mu \mathrm{mol} / \mathrm{L}, 0.50$ $\mu \mathrm{mol} / \mathrm{L}$, or $1.00 \mu \mathrm{mol} / \mathrm{L}$ for $48 \mathrm{hrs}$ in complete growth medium, or add ANGPTL8 gene plasmids transfection with lipofectamin2000, or $2 \mathrm{pmol} / \mathrm{L}$ ANGPTL8 peptide (Phoenix Pharmaceuticals, Burlingame, CA, USA) to a dish and were subjected to immunofluorescent staining.

\section{ELISA for detecting plasma ANGPTL8}

Fasting venous blood was collected in EDTA tubes from the tail vein, and plasma lipids were measured using standard enzymatic assays. Human ANGTPL8 was measured using ELISA (Phoenix Pharmaceuticals, Burlingame, CA, USA).

\section{RNA isolation and quantitative RT-PCR analysis}

Total RNA was isolated from $100 \mathrm{mg}$ of heart using the RNeasy mini kit (Qiagen). Real-time quantitative RTPCR (qRT-PCR) analysis was performed on an ABI 7700 Sequence Detector (Applied Biosystems, Grand Island, NY, USA) using SYBR Green (RT2 SYBR Green qPCR Kit; Qiagen, Boston, MA, USA). Data were normalized to the expression of housekeeping genes (as an endogenous control). Changes in gene expression were normalized to 
control liver and cardiac muscle samples.

\section{Western blotting}

Total protein extracts from heart tissue were evaluated with a Cytoplasmic and Nuclei Extraction Kit (Thermo Scientific, Rockford, IL, USA). Protein concentrations were determined using the BCA-200 Protein Assay kit (Pierce, Grand Island, NY, USA); equal amounts of protein were separated by SDS-PAGE to nitrocellulose membranes and incubated with primary antibodies anti ANGPTL8 (1:1,000 dilutions), antiPirB(1:2000 dilution), anti ISL-1 (1:1000 dilution), anti PHH3 (1:2000 dilution) and anti-actin (1: 2,000 dilutions) and anti-Lamin (1:2000 dilution). Horseradish peroxidase secondary antibodies were used, and chemiluminescence was determined using the SuperSignal West Dura detection system (Pierce); Cytoplasmic marker (actin) and nuclei marker (lamin) were used to confirm equal loading. All Western blots were performed in duplicate.

\section{Echocardiography}

Echocardiographic measurements of LV mass, fractional shortening and LV posterior wall thickness were made from digital images acquired with a $12 \mathrm{MHz}$ broadband transducer (S12 probe, Philips Ultrasound, Bothell, WA) in M-mode under 2D echo short axis view. LV mass $=1.05[\text { LVIDd +IVS+LVIPW }]^{3}-[\text { LVIDd }]^{3}$. Fractional shortening was evaluated from the following formula: FS=(LVIDd-LVIDs)/LVIDd X 100.

\section{Data analysis}

Data were analysed using Statview software (SAS, Cary, NC, USA). The values are presented as mean \pm SEM. Differences were analyzed by repeated measures ANOVA with Fisher's post hoc test and were considered significant at $\mathrm{p}<0.05$.

\section{Supplementary material} online.

Supplementary material is available at Oncotarget

\section{ACKNOWLEDGMENTS}

SC and PAG were responsible for the conception and design of the study, acquisition and analysis of the data and drafting of the article. SC and JC performed data acquisition and analysis and contributed to writing the manuscript. JS, XM, PH, JH, ZP, and NHM made substantial contributions to the conception of the study and critically revised the manuscript. PAG is the guarantor of this work and as such had full access to all the data in the study and takes responsibility for the integrity of the data and the accuracy of the data analysis. All listed authors approved the final version of the manuscript.

\section{CONFLICTS OF INTEREST}

The authors declare that there is no conflict(s) of interest associated with this manuscript.

\section{FUNDINGS}

This work was supported by the Mark and Mary Alice Shepherd Fund of The Baylor Foundation (PAG) and The National Natural Science Funds of China (81420108018) (PH).

\section{Editorial note}

This paper has been accepted based in part on peerreview conducted by another journal and the authors' response and revisions as well as expedited peer-review in Oncotarget.

\section{REFERENCES}

1. Swain SM, Whaley FS, Ewer MS. Congestive heart failure in patients treated with doxorubicin: a retrospective analysis of three trials. Cancer. 2003; 97:2869-79.

2. Lee V, Randhawa AK, Singal PK. Adriamycin-induced myocardial dysfunction in vitro is mediated by free radicals. Am J Physiol. 1991; 261:H989-995.

3. Gille L, Nohl H. Analyses of the molecular mechanism of adriamycin-induced cardiotoxicity. Free Radic Biol Med. 1997; 23:775-782.

4. Bristow MR, Sageman W, Scott R, et al. Acute and chronic cardiovascular effects of doxorubicin in the dog: the cardiovascular pharmacology of drug-induced histamine release. J Cardiovasc Pharmacol . 1980; 2:487-515.

5. Monnet E, Chachques JC. Animal Models of Heart Failure: What Is New? Ann Thorac Surg. 2005; 79:1445-1453.

6. Zhang S, Liu X, Bawa-Khalfe T, Lu LS, Lyu YL, Liu LF, Yeh ET. Identification of the molecular basis of adriamycininduced cardiotoxicity. Nat Med. 2012; 18:1639-42.

7. Senyo SE, Steinhauser ML, Pizzimenti CL, Yang VK, Cai L, Wang M, Wu TD, Guerquin-Kern JL, Lechene CP, Lee RT. Mammalian heart renewal by pre-existing cardiomyocytes. Nature. 2013; 493:433-436.

8. Eulalio A, Mano M, Dal Ferro M, Zentilin L, Sinagra G, Zacchigna S, Giacca M. Functional screening identifies miRNAs inducing cardiac regeneration. Nature. 2012; 492:376-381.

9. Smart N, Bollini S, Dubé KN, Vieira JM, Zhou B, Davidson S, Yellon D, Riegler J, Price AN, Lythgoe MF, 
Pu WT, Riley PR. De novo cardiomyocytes from within the activated adult heart after injury. Nature. 2011; 474:640644.

10. Bolli R, Chugh AR, D'Amario D, Loughran JH, Stoddard MF, Ikram S, Beache GM, Wagner SG, Leri A, Hosoda T, Sanada F, Elmore JB, Goichberg P, et al. Cardiac stem cells in patients with ischaemic cardiomyopathy (SCIPIO): initial results of a randomised phase 1 trial. Lancet. 2011; 378:1847-1857.

11. Beltrami AP, Barlucchi L, Torella D, Baker M, Limana F, Chimenti S, Kasahara H, Rota M, Musso E, Urbanek K, Leri A, Kajstura J, Nadal-Ginard B, et al. Adult cardiac stem cells are multipotent and support myocardial regeneration. Cell. 2003; 114:763-776.

12. Jopling C, Sleep E, Raya M, Martí M, Raya A, Izpisúa Belmonte JC. Zebrafish heart regeneration occurs by cardiomyocyte dedifferentiation and proliferation. Nature. 2010; 464:606-609.

13. Porrello ER, Mahmoud AI, Simpson E, Hill JA, Richardson JA, Olson EN, Sadek HA. Transient regenerative potential of the neonatal mouse heart. Science. 2011; 331:1078-1080.

14. Song K, Nam YJ, Luo X, Qi X, Tan W, Huang GN, Acharya A, Smith CL, Tallquist MD, Neilson EG, Hill JA, Bassel-Duby R, Olson EN. Heart repair by reprogramming non-myocytes with cardiac transcription factors. Nature. 2012; 485:599-604.

15. Qian L, Huang Y, Spencer CI, Foley A, Vedantham V, Liu L, Conway SJ, Fu JD, Srivastava D. In vivo reprogramming of murine cardiac fibroblasts into induced cardiomyocytes. Nature. 2012; 485:593-598.

16. Nowbar AN, Mielewczik M, Karavassilis M, Dehbi HM, Shun-Shin MJ, Jones S, Howard JP, Cole GD, Francis DP; DAMASCENE writing group. Discrepancies in autologous bone marrow stem cell trials and enhancement of ejection fraction (DAMASCENE): weighted regression and metaanalysis. BMJ. 2014; 348:g2688.

17. Laugwitz KL, Moretti A, Lam J, Gruber P, Chen Y, Woodard S, Lin LZ, Cai CL, Lu MM, Reth M, Platoshyn O, Yuan JX, Evans S, et al. Postnatal isl1+ cardioblasts enter fully differentiated cardiomyocyte lineages. Nature. 2005;433:647-653.

18. Moretti A, Caron L, Nakano A, Lam JT, Bernshausen A, Chen Y, Qyang Y, Bu L, Sasaki M, Martin-Puig S, Sun Y, Evans SM, Laugwitz KL, et al. Multipotent embryonic isl1+ progenitor cells lead to cardiac, smooth muscle, and endothelial cell diversification. Cell. 2006;127:1151-1165.

19. Di Felice V, Zummo G. Stem cell populations in the heart and the role of Isl1 positive cells. Eur J Histochem. 2013; 57:e14.

20. Masters M, Riley PR. The epicardium signals the way towards heart regeneration. Stem Cell Res. 2014; 13: 683692.

21. Smart N, Paul R. Riley PR. The epicardium as a candidate for heart regeneration. Future Cardiol. 2012; 8: 53-69.
22. Zhang R. Lipasin, a novel nutritionally-regulated liverenriched factor that regulates serum triglyceride levels. Biochem. Biophys. Res. Commun. 2012; 424: 786-792.

23. Fu Z, Yao F, Abou-Samra AB, Zhang R. Lipasin, thermoregulated in brown fat, is a novel but atypical member of the Angiopoietin-like protein family. Biochem. Biophys. Res. Commun. 2013; 430: 1126-1131.

24. Zhang R, Abou-Samra AB. Emerging roles of Lipasin as a critical lipid regulator. Biochem Biophys Res Commun. 2013; 432: 401-405.

25. Ren G, Kim JY, Smas CM. Identification of RIFL, a novel adipocyte-enriched insulin target gene with a role in lipid metabolism. Am. J. Physiol. Endocrinol. Metab. 2012; 303: E334-E351.

26. Quagliarini F, Wang Y, Kozlitina J, Grishin NV, Hyde R, Boerwinkle E, Valenzuela DM, Murphy AJ, Cohen JC, Hobbs HH. Atypical Angiopoietin-like protein that regulates ANGPTL3. Proc. Natl. Acad. Sci. USA. 2012; 109: 19751-19756.

27. Wang Y, Quagliarini F, Gusarova V, Gromada J, Valenzuela DM, Cohen JC, Hobbs HH. Mice lacking ANGPTL8 (Betatrophin) manifest disrupted triglyceride metabolism without impaired glucose homeostasis. Proc Natl Acad Sci U S A. 2013; 110: 16109-16114.

28. Yi P, Park JS, Melton DA. Betatrophin: a hormone that controls pancreatic beta cell proliferation. Cell. 2013; 153: 747-758.

29. Yi P, Park JS, Melton DA. Perspectives on the activities of ANGPTL8/betatrophin. Cell. 2014; 159:467-468.

30. Chen J, Chen S, Huang P, Meng XL, Clayton S, Shen JS, Grayburn PA. In vivo targeted delivery of ANGPTL8 gene for beta cell regeneration in rats. Diabetologia. 2015; 58:1036-1044.

31. Chen S, Ding JH, Bekeredjian R, Yang BZ, Shohet RV, Johnston SA, Hohmeier HE, Newgard CB, Grayburn PA. Efficient gene delivery to pancreatic islets with ultrasonic microbubble destruction technology. Proc Natl Acad Sci U S A. 2006; 103: 8469-8474.

32. Chen S, Ding JH, Grayburn PA. Reversal of streptozotocininduced diabetes in rats by gene therapy with betacellulin and pancreatic duodenal homeobox-1. Gene therapy. 2007; 14: 1102-1110.

33. Chai RJ, Chen S, Ding J, Grayburn P. Efficient, glucose responsive and islet-specific transgene expression by a modified rat insulin promoter. Gene Therapy. 2009; 16: 1202-1209.

34. Chen S, Shimoda M, Wang MY, Ding J, Noguchi H, Matsumoto S, Grayburn PA. Regeneration of pancreatic islets in vivo by ultrasound-targeted gene therapy. Gene Therapy. 2010; 17: 1411-1420.

35. Shimoda M, Chen S, Noguchi H, Matsumoto S, Grayburn PA. In vivo non-viral gene delivery of human vascular endothelial growth factor improves revascularisation and restoration of euglycaemia after human islet transplantation 
into mouse liver. Diabetologia. 2010; 53: 1669-1679.

36. Chen S, Shimoda M, Chen J, Matsumodo S, Grayburn PA. Transient overexpression of cyclin D2/CDK4/GLP1 genes induces proliferation and differentiation of adult pancreatic progenitors and mediates islet regeneration. Cell Cycle. 2012; 11: 695-705.

37. Chen S, Shimoda M, Chen J, Matsumoto S, Grayburn PA. Ectopic transgenic expression of NKX2.2 induced differentiation of adult pancreatic progenitors and mediates islet regeneration. Cell Cycle. 2012; 11: 1544-1553.

38. Zheng J, Umikawa M, Cui C, Li J, Chen X, Zhang C, Huynh H, Kang X, Silvany R, Wan X, Ye J, Cantó AP, Chen $\mathrm{SH}$, et al. Inhibitory receptors bind ANGPTLs and support blood stem cells and leukaemia development. Nature. 2012; 485:656-660.

39. Atwal JK, Pinkston-Gosse J, Syken J, Stawicki S, Wu Y, Shatz C, Tessier-Lavigne M. PirB is a functional receptor for myelin inhibitors of axonal regeneration. Science. 2008; 322:967-970.

40. Balmer GM, Bollini S, Dubé KN, Martinez-Barbera JP, Williams O, Riley PR. Dynamic haematopoietic cell contribution to the developing and adult epicardium. Nat Commun. 2014; 5: 4054.

41. Deng M, Lu Z, Zheng J, Wan X, Chen X, Hirayasu K, Sun H, Lam Y, Chen L, Wang Q, Song C, Huang N, Gao GF, et al. A motif in LILRB2 critical for Angpt12 binding and activation. Blood. 2014; 124:924-35.

42. Zhang CC, Kaba M, Iizuka S, Huynh H, Lodish HF. Angiopoietin-like 5 and IGFBP2 stimulate ex vivo expansion of human cord blood hematopoietic stem cells as assayed by NOD/SCID transplantation. Blood. 2008; 111:3415-3423.

43. Zhang CC, et al. Angiopoietin-like proteins stimulate ex vivo expansion of hematopoietic stem cells. Nat Med. 2006; 12:240-245.

44. Kitamura T, Kitamura YI, Funahashi Y, Shawber CJ, Castrillon DH, Kollipara R, DePinho RA, Kitajewski J, Accili D. A Foxo/Notch pathway controls myogenic differentiation and fiber type specification. J Clin Invest. 2007; 117:2477-85.

45. Lin MI, Price EN, Boatman S, Hagedorn EJ, Trompouki E, Satishchandran S, Carspecken CW, Uong A, DiBiase A, Yang S, Canver MC, Dahlberg A, Lu Z, et al. Angiopoietinlike proteins stimulate HSPC development through interaction with notch receptor signaling. Elife. 2015; 4.

46. Rizzo P, Mele D, Caliceti C, Pannella M, Fortini C, Clementz AG, Morelli MB, Aquila G, Ameri P, Ferrari R. The role of notch in the cardiovascular system: potential adverse effects of investigational notch inhibitors. Front Oncol. 2015; 4:384.

47. Iliskovic N, Singal PK. Lipid Lowering: An Important Factor in Preventing Adriamycin-Induced Heart Failure. American Journal of Pathology.1997; 150: 727-734.

48. Chen S, Shohet RV, Bekeredjian R, Frenkel P, Grayburn
PA. Optimization of ultrasound parameters for cardiac gene delivery of adenoviral or plasmid deoxyribonucleic acid by ultrasound-targeted microbubble destruction. J Am Coll Cardiol. 2003; 42:301-308.

49. Cary LC, Goebel M, Corsaro BG, Wang HG, Rosen E, Fraser MJ. Transposon mutagenesis of baculoviruses: analysis of Trichoplusia ni transposon IFP2 insertions within the FP-locus of nuclear polyhedrosis viruses. Virology.1989; 172:156-169.

50. Yusa K, Zhou L, Li MA, Bradley A, Craig NL. A hyperactive piggyBac transposase for mammalian applications. Proc Natl Acad Sci USA. 2011; 108:15311536.

51. Claycomb WC, Lanson NA Jr, Stallworth BS, Egeland DB, Delcarpio JB, Bahinski A, Izzo NJ Jr. HL-1 cells: A cardiac muscle cell line that contracts and retains phenotypic characteristics of the adult cardiomyocyte. Proc Natl Acad Sci USA. 1998; 95:2979-2984. 